\title{
Modified fixation free plug technique using a new 3D multilamellar implant for inguinal hernia repair
}

\author{
A retrospective study of a single operator case series
}

\author{
G. Amato - A. Agrusa - G. Romano • \\ G. Cocorullo • G. Di Buono - S. Mularo • \\ G. Gulotta
}

Received: 11 February 2012/Accepted: 26 April 2013/Published online: 8 May 2013

(C) Springer-Verlag France 2013

\begin{abstract}
Aim Implant fixation, mesh shrinkage and poor quality of tissue ingrowth are unresolved issues in modern hernia repair. Many complications reported in the literature such as bleeding, nerve entrapment, hematoma, pain, discomfort, and testicular complications, are considered to be a direct results of implant fixation. This article describes the outcomes of a procedure carried out using a handcrafted implant that addresses the issues consequent to point fixation.

Methods This was a retrospective study on the short, medium and long-term results of placing a-modified, fixation free three-dimensional polypropylene implant in 61 patients who underwent inguinal hernia repair using a novel delivery technique. The follow up length was at least 36 months postop.

Results Only minor adverse events and a low complication rate of the procedure were observed in this patient sample. There were no long- term complications. Postoperative pain was very low in both the short and long term. No chronic pain was reported. No recurrences occurred.
\end{abstract}

Electronic supplementary material The online version of this article (doi:10.1007/s10029-013-1102-y) contains supplementary material, which is available to authorized users.

G. Amato $(\bowtie) \cdot$ A. Agrusa - G. Romano - G. Cocorullo ·

G. Di Buono - G. Gulotta

Department of General Surgery, Urgency and Organ

Transplantation, University of Palermo, Via Rapisardi 66,

90144 Palermo, Italy

e-mail: amatomed@gmail.com

S. Mularo

Department of Anesthesia and Intensive Care, University of

Palermo, Palermo, Italy
Conclusion The results of this retrospective study on a new method of inguinal hernia repair using a three dimensional handcrafted multilamellar implant delivered with a modified placement technique are promising. The short-, medium- and long-term complications were notably low. No recurrences were noticed but, more importantly, no chronic pain and extremely low discomfort rates were observed even in the long term.

Keywords Hernia - Inguinal · Herniorrhaphy · Prostheses and implants · Prosthesis fixation

\section{Introduction}

Inguinal hernia repair is one of the most frequently performed surgical procedures. In the US alone, more than 800,000 hernia repairs are performed every year [1]. Several decades after Bassini [2] developed the first "pure tissue repair" technique, the modern era of inguinal hernia repair began in 1959 with Usher, who first used a synthetic flat mesh to cover an inguinal hernia opening [3]. Lichtenstein [4] expanded the use of polypropylene (PP) flat mesh and developed a new concept for outpatient hernia repair: the so-called "tension-free hernia repair". In the last few decades, prosthetic hernia techniques have been further implemented through the use of static plugs or other static three-dimensional (3D) structures to "fill" the hernia defect. PP is currently the most frequent used material for open prosthetic inguinal hernia repair [3]. Undoubtedly innovations have been important; because current techniques are based on the anatomical pathology and not the underlying cause, it seems that a true "repair" has really been performed, however, only an attempt at anatomical reinforcement has occurred. It should also be considered 
that reinforcement with static implants merely creates a physical and non-functionally integrated barrier that often shrinks and regresses over time because of localized tissue reaction. This probably contributes to the disappointingly high rate of complications such as recurrence and, especially, pain and discomfort. Regardless of the constant improvement in surgical technique and materials, hematomas, bleeding, nerve entrapment, testicular damage, and recurrences remain issues [5, 6]. By examining the literature, and drawing upon more than 20 years of surgical experience, it appears evident that the short-, medium- and long-term complications are still unacceptably high. Deep fixation is well accepted as a cause of many of the common complications, including chronic pain [5-8]: thus the authors' primary goal was to use the geometry of the implant itself as a means to eliminate fixation. Starting with these considerations, it was envisaged that implant shape and structure could be used to eliminate the need to fix implant. This was eventually realized using the flexible properties of PP to form a multilamellar "flower"-shaped design similar to a radial spring. In conjunction with correct sizing, this ensures that the implant is always bigger than the defect, thus gripping the inside of the defect.

This study involved 61 patients who underwent hernia repair with the above-described implant. Data gathered from this study were used to evaluate a modified plug technique to eliminate the need for fixation.

\section{Materials and methods}

\section{Overview}

This study was approved by the ethics committee of the University Palermo. Between June 2005 and July 2008, 61 patients underwent inguinal hernia repair and were retrospectively analyzed at different time until they reached the 36 months postoperative period.

The primary objective of this retrospective study was to evaluate the effectiveness and long-term complication rates of a modified PP implant. The implant was inserted with a modified delivery technique using a set of differently sized steel tubes to dilate the tissues prior to delivering the implant. The tubes were used to constrain the implant into a tight shape, and the implant would then spring open once inside the defect. In all patients, no additional anterior flat mesh was placed to cover the hernia defect. If local/ regional anesthesia was used to perform the repair, the patient was invited to cough after delivering the implant in order to demonstrate that the implant remained within the hernia defect.

During the early postoperative period, the patients were evaluated at 3, 7 and 15 days. Procedural complications up

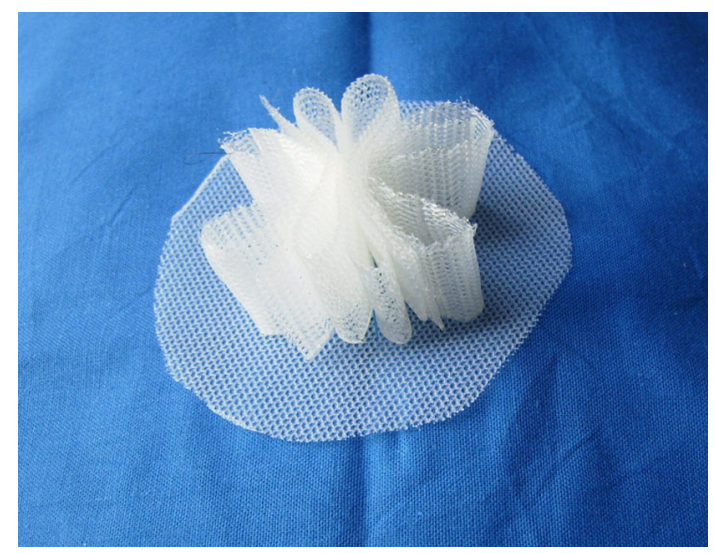

Fig. 1 The handcrafted 3D implant made by folded polypropylene mesh connected with a flat circular mesh intended to face the peritoneal sheath

Table 1 Post procedure evaluations (from end of procedure to discharge)

\begin{tabular}{l}
\hline Early postoperative evaluation \\
\hline Freedom from recurrence \\
Infection/abscess \\
Dislodgement of the implant \\
Discomfort from the implant \\
Nerve pain/problems \\
Hematoma \\
Seroma \\
Testicular swelling \\
Wound complications \\
Length of stay (days) \\
Other complications/adverse events
\end{tabular}

to 30 days after surgery or at hospital discharge, whichever occurred later, were evaluated and reported. The patients were questioned about postoperative pain after 1, 12, and 36 months postoperatively. Long-term complications were also evaluated and reported up to 36 months postoperatively.

Implant design and procedure

The new hernia repair is characterized by a dual system that involves the use of a synthetic and permanent PP implant (Fig. 1) and a reusable dilation and deployment tool made from stainless steel and plastic tubing. Each implant was hand-made from commercially available strips of PP and fashioned during the surgical procedure using one or two $2 / 0$ prolene sutures at its central aspect to create a multilamellar shape. Attached to the core was a flat PP disk that when implanted preperitoneally helped to protect the repair and stabilize the device. The implants were sewn into different sizes according to the width of the hernia 
Table 2 Post procedure evaluations (from discharge to final followup at 36 months)

Evaluation at 2 weeks, 1, 6, 12 and 36 months

Freedom from recurrence

Pain assessment through VAS pain score (1-10)

Infection/abscess

Dislodgement of the implant

Discomfort from the implant (subjective evaluation)

Hematoma

Seroma

Testicular swelling-atrophy-orchitis

Adverse events

defect. The size of the handcrafted prosthesis was designed to be $30 \%$ larger than the width of the hernia opening. The circumference of the disk varied from 5 to $7.5 \mathrm{~cm}$ depending on the hernia size and, consequently, on the dimension of the lamellar core of the implant to be delivered.

\section{Study design and endpoints}

This investigation was a retrospective study that was designed to collect perioperative and postoperative data to evaluate the new hernia system in terms of the ability of the device to be delivered and then repair indirect, direct and mixed hernia as well as primary and recurrent inguinal hernia defects. Data from the perioperative period (Table 1) and long-term (up to 36 month) follow-up (Table 2) were collected to assess the 36 month efficacy and, importantly, adverse events.

All 61 patients were analyzed to evaluate the primary and secondary endpoints. Follow-up of all patients was conducted by in office visit on all patients at 15 days and 1, 6, 12, and 36 months. A telephone call substituted the office visit if the patient could not attend the clinical examination.

Patient eligibility

\section{Confidentiality}

All clinical information obtained in the study was considered to be confidential and was used only for research purposes. The identity of individual subjects was kept confidential to the extent permitted by the applicable laws and regulations and safe medical practice.

\section{Inclusion criteria}

- Scheduled to undergo routine inguinal hernia repair

- Competent to give consent
- Clinically relevant inguinal hernia (classification: indirect, direct, mixed)

- Male or female

- Age $\geq 18-85$ years

- Life expectancy of at least 12 months

- Diagnosed with direct, indirect or mixed inguinal hernia, unilateral or bilateral

- Primary or recurrent hernia

\section{Exclusion criteria}

- Signs of obvious local or systemic infection

- Hernia was not in the inguinal area (e.g., spigelian or femoral hernia)

- Presenting with unstable angina or NYHA class of IV

- Pregnant

- Active drug user

- Immunosuppression, prednisone treatment of $>15 \mathrm{mg} /$ day, active chemotherapy

- End stage renal disease

- Abdominal ascites

- Skin infection in area of surgical field

- $\quad$ BMI > 35

\section{Surgical method}

Patients underwent hernia repair by the Physio Hernia Repair (PHR) technique using the hand-made PP flowershaped implants and above-described delivery method. Antibiotic prophylaxis was administered according to institutional guidelines. General, local or regional anesthesia was administered at the physician's discretion.

The following standardized PHR technique was performed in all patients:

A. A 4-8 cm skin incision in the inguinal area was made.

B. Dissection was performed through Scarpa's fascia, to the external oblique aponeurosis.

C. Exposure was gained to the external inguinal ring and external oblique aponeurosis.

D. The external oblique aponeurosis was opened and the cord was dissected, and elevated, defining the hernia sac location and internal ring.

E. For indirect hernias, removal of adhesions and scar tissue around the internal inguinal ring, dissection of the sac, high ligation and excision of sac were performed.

F. Before releasing the stump into the abdominal cavity, finger dissection of the parietal peritoneum from the posterior abdominal wall was performed. The width of dissection was appropriate to achieve a preperitoneal free space of ca. $6 / 7 \mathrm{~cm}$ in diameter, thus it was large 

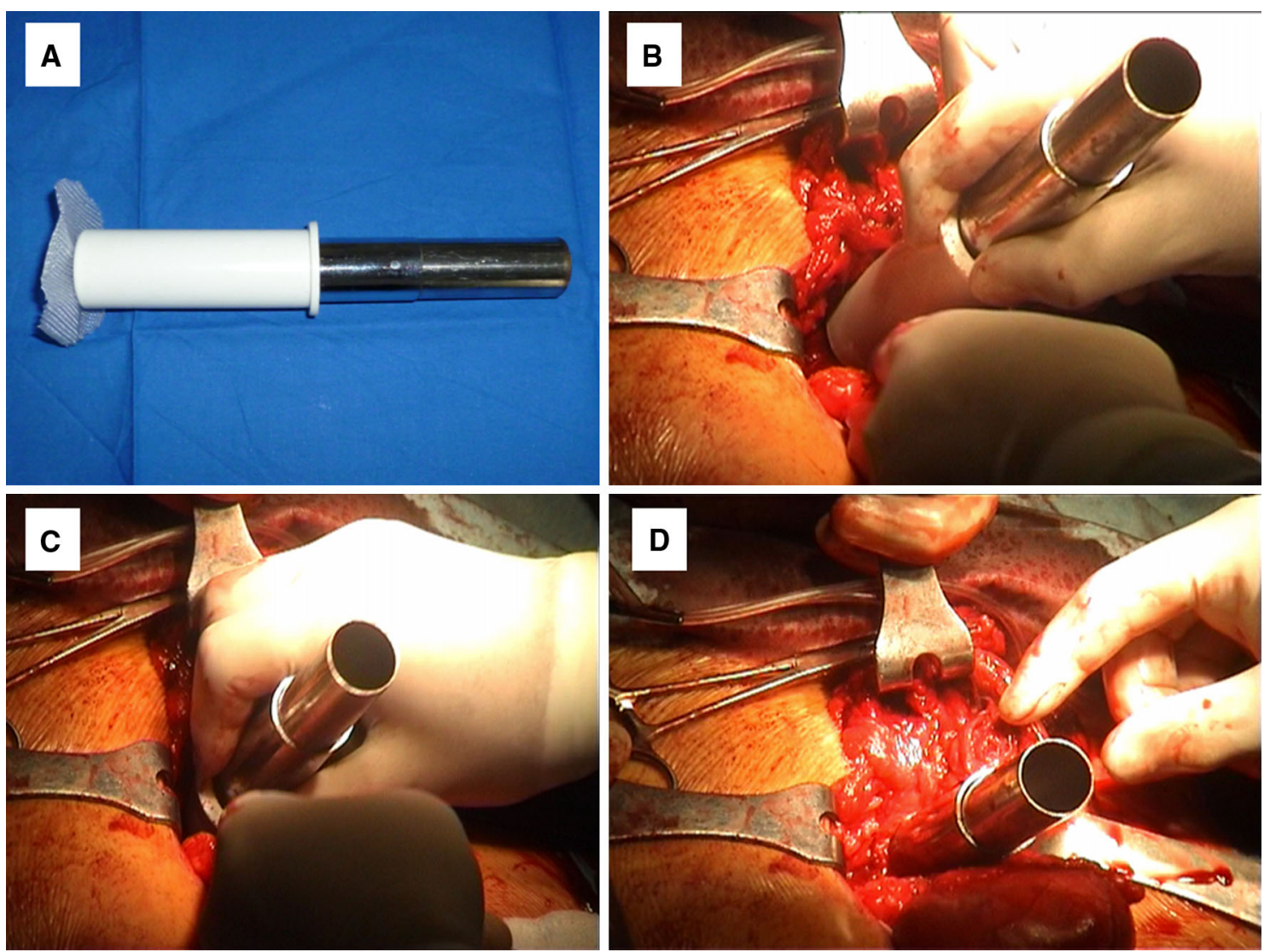

Fig. 2 Tools and delivery mode. a The delivery tool loaded with the implant. The external tube holds two other tubes. The preperitoneal disk lies outside of the complex to be placed beyond the hernia frame. The 3D multilamellar implant is placed within the second tube. b Insertion of the tube system containing the implant within the hernia opening The external tube is wider than the hernia defect and dilates

enough to allow the preperitoneal disk of the implant to cover the posterior aspect of Hesselbach's triangle.

G. For direct hernias, a dissection of the sac was performed from the inguinal structures until the transversalis fascia, followed by removal of adhesions and scar tissue around the hernia opening. Finger dissection of the transversalis fascia from the transverse muscle was then performed to accommodate placement of the preperitoneal disk of the implant (as appropriate). When this finger guided maneuver was not achievable, the transversalis fascia was breached to allow for placement of the implant disk in the preperitoneal space facing the peritoneal sheath.

H. According to hernia size, the implant was crafted by cutting from large mesh strips of polypropylene ca. $1.5-2 \mathrm{~cm}$ in height and having ca. $8-10 \mathrm{~cm}$ length. The strips, folded in two or three parts in their longitudinal aspect, were connected at its center with one or two prolene stitches. The prosthetic structure was completed by sewing with a single stitch at one

the muscular edge. $\mathbf{c}$ Tube system inserted within the hernia frame. The second tube contains the implant. d After dilation of the hernia opening the delivery tube has been removed. The inner tube act as a plunger to eject the implant outside the tubing system into the hernia defect

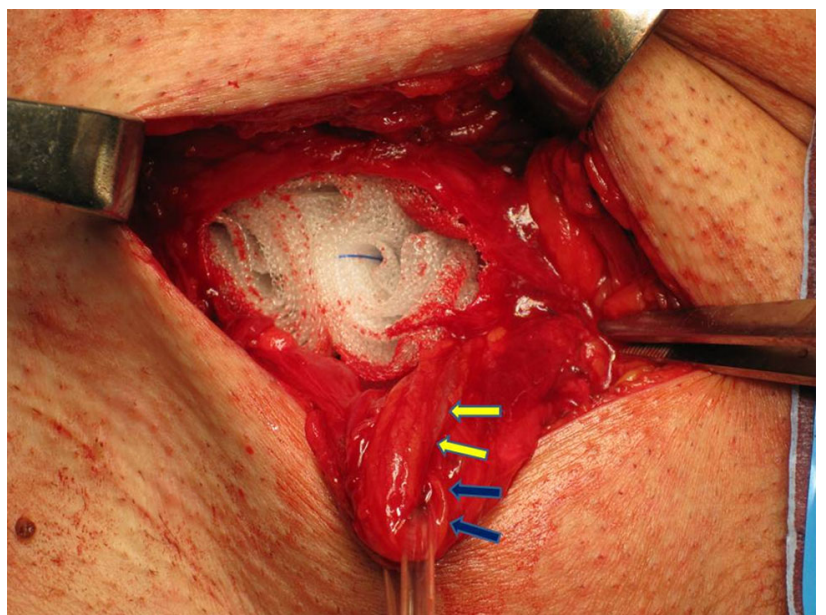

Fig. 3 Indirect hernia: the implant fully obliterates the hernia opening without fixation The spermatic cord structures are not compressed by the lamellas. Both, spermatic vein (yellow arrow) and spermatic duct (blue arrow) are clearly visible. The blue colored stitch in the middle of the implant is the suture that connects the folded structure of the implant 


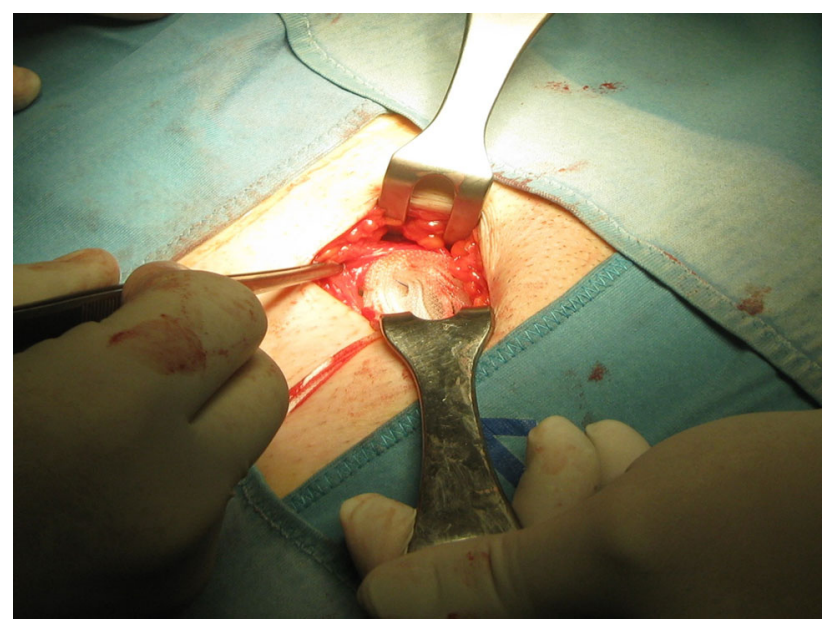

Fig. 4 Direct hernia: obliteration of the hernia defect. Note how the lamellas of the implant are compressed by the myotendineal structure of the fossa inguinalis media. The inherent centrifugal expansion of the flower-shaped prosthesis within the hernia defect completely fills the gap

bottom a circular shaped mesh, which dimension varied from 5 to $7.5 \mathrm{~cm}$ in diameter

I. The implant was compressed and loaded into the tube system (Fig. 2a).

J. The steel tube system was then inserted into the hernia defect to allow for delivery of the flower-shaped inguinal hernia implant (Fig. 2b) and gentle dilation of the inguinal opening (Fig. 2c).

K. The external tube of the system was then pulled back, taking care that the preperitoneal disk remained beyond the hernia opening (Fig. 2d).

L. The implant was deployed by pushing the inner tube, releasing the constrained inguinal hernia implant within the hernia opening (Fig. 3).

M. After delivery, the implant fully obliterated the defect of both indirect (Fig. 3) and direct hernias. (Fig. 4) If needed, forceps-guided adjustment of the inguinal hernia implant was performed to allow for an adequate flat placement of the implant in the preperitoneal space.

N. No additional flat mesh was placed to cover the hernia opening.

O. Closure of the external oblique fascia was performed with absorbable sutures. Skin closure was performed with a subdermal technique.

P. Where possible wound drains were avoided.

Patients were discharged at the discretion of the physician, and the length of stay was noted.

Data and statistical analysis

In general, the statistics for continuous variables included mean, median, and standard deviation. Binary variables were described with numbers and percentages.
The clinical investigator ensured that the protocol requirements for data mining were met and that all data collected were accurate. Master data records and patient notes are held by the investigators.

\section{Additional information}

At the time of this writing, the longest follow-up of any patient in the 61 patient group was 81 months (6.75 years). Although the analysis was only conducted to the end point of 36 months, $65 \%$ of patients are now out beyond 5 years.

\section{Results}

A total of 61 consecutive patients who underwent the procedure between January 2005 and July 2008 were retrospectively reviewed. The group comprised 59 (97\%) male and 2 female patients (3\%). The median BMI value was 29.38 (range 23-34). Median operative time was $32.43 \mathrm{~min}$ (range $21-53 \mathrm{~min}$ ).

Patients were followed up to the end point of 36 months. The median age of those patients was 53.25 years (range 18-86 years). All patients were clinically examined at 2 weeks and 1, 6, 12 and 36 months with few exceptions during the follow-up. On these few occasions, the clinical examination was achieved at the next scheduled evaluation. Only three patients could not attend the 36 months clinical evaluation. In these cases, a telephone call confirmed the absence of complications and recurrences. One patient died of heart failure 14 months after the procedure, thus data analysis was only undertaken to 60 patients for the 36-month follow-up period.

Twenty-seven patients (44\%) underwent outpatient surgery. Thirty-two patients were discharged the day following the procedure. Only two patients $(3 \%)$ were discharged on the second postoperative day because of nonsurgically related adverse events (one developed urinary retention following prostate hypertrophy, and the other developed orthostatic low pressure following spinal anesthesia). The median length of stay was 0.92 days (range 0.5-3 days).

The following hernia types were recorded: right indirect $22(35.2 \%)$, left indirect $15(23.8 \%)$, right direct 3 $(4.7 \%)$, left direct $4(6.2 \%)$, recurrent left $9(14.3 \%)$, recurrent right $5(8 \%)$, left mixed $1(1.5 \%)$, right mixed 4 $(6.2 \%)$.

No short- or long-term recurrence was reported. In terms of surgically related complications no infections occurred. There were one hematoma, three seromas and three cases of testicular swelling (all from large inguinoscrotal hernias) with no testicular damage (Table 3). 
Table 3 Perioperative complications

\begin{tabular}{llll}
\hline Complication & Number & $\%(n=61)$ & Comments \\
\hline Hematoma & 0 & 0 & \\
Swelling of the scrotal skin & 3 & 4.9 & $\begin{array}{c}\text { All resolved, all related to large } \\
\text { inguinal scrotal hernias (no } \\
\text { testicular damage) }\end{array}$ \\
Pain not controlled by usual analgesics & 0 & 0 & \\
Bleeding & 0 & 0 & \\
Failure to deploy device & 0 & 0 & \\
\hline
\end{tabular}

Table 4 Early complications within 2 weeks postoperative

\begin{tabular}{llll}
\hline Complication & Number & $\%(n=61)$ & Comments \\
\hline Recurrence & 0 & 0 & \\
Infection/abscess & 0 & 0 & \\
Dislodgement of the implant & 0 & 0 & \\
$\begin{array}{l}\text { Discomfort from the implant } \\
\text { Nerve pain/problems }\end{array}$ & 0 & 0 & Subjective assessment by the patient through VAS pain score (1-10) \\
$\begin{array}{l}\text { Hematoma } \\
\text { Seroma }\end{array}$ & 1 & 0 & Subjective assessment by the patients through VAS pain score (1-10) \\
$\begin{array}{l}\text { Swelling of the scrotal skin } \\
\text { Wound complications }\end{array}$ & 3 & 4.9 & Patient resolved without drain \\
$\begin{array}{l}\text { Other complications } \\
\text { Other complications }\end{array}$ & 0 & 0 & All resolved, all related to large inguinal scrotal hernias (no atrophy or loss) \\
Adverse events requiring further & 0 & 1.6 & Discharge delay for bladder training due to urinary retention caused by \\
procedure & 1 & 1.6 & Discharge delay due to orthostatic low pressure following spinal anesthesia \\
\hline
\end{tabular}

a All patients resolved by the 1-month follow-up

There were no long-term complications. The postoperative pain scores, as measured by a visual analog scale (VAS), were very low in the first month: only four patients had a score of $>3$. In all patients the pain score diminished to 0 by the third month and beyond. No chronic pain was reported in any patient. In addition, subjective discomfort was virtually absent in all patients beyond 6 months postoperatively (Table 4). Of note, two patients had bilateral hernias bringing the total number of implants to 63 . These patients underwent placement of one implant each groin.

\section{Discussion}

The introduction of modern materials, such as PP, for the repair of inguinal hernia has allowed for the development of "less tension" techniques. This term is being used with respect to low tension during surgical implant placement. However, the authors feel that the use of the term "tension free" can only be applied to the moment of implantation if any form of fixation (sutures, tackers, or glues) is used to fix one set of tissues to the other-using the implant as a "bridge" or reinforcement. Non-physiologic fixation goes against the principles of the dynamic nature of the inguinal region $[9,10]$.

Shrinkage of the implant often occurs due to poor quality scar formation and can lead to weak spots which may in turn lead to recurrence or induce tension at any of the fixed points used during the initial surgery. This may even occur with absorbable materials, which still induce a "point" scar.

Through years of cadaveric studies and animal work, the authors hypothesized that a new type of implant would potentially eliminate point fixation and improve scar tissue formation within the implant. The implant and delivery method described in this study were a result of that hypothesis. The concept of this new implant design was that by its unique 3D geometry combined with a compressed state, it would translate expulsion forces into lateral gripping forces. The pre-dilation of the hernia defect helps to augment the gripping action of the muscles and simplify insertion of the prosthesis. Recently published animal studies describe the nature of the implant and its histological impact [9]. 
This retrospective study aimed to collate the data from patients who underwent the herein described surgery with a minimum follow-up of 36 months. This time frame was chosen because it seemed able to capture any short-, medium- and long-term complications related the procedure and device.

There are marked differences between this modified repair and normal plug repairs. The use of a delivery device to constrain the implant prior to delivery ensures a constant radial expansion post-delivery. The "flower" shape of the device has inherent outward recoil as opposed to cone shaped plugs, which have a tendency to collapse. The dilation of the inguinal opening is seen as a critical step in divulsing any blocked tissues that demonstrate adhesions or fibrosis [11-14] and restoring physiologic movement which allows for a gripping motion [15].

The present results show that the dilation and delivery of the device using the tube system is safe and causes no complications related to this new and important procedural step. These results show that there are no complications related to damage or compression of the spermatic cord (Fig. 3). The lack of this compression has been reported in human cases [16] and has been histologically verified in animal studies [9].

The results of this study appear to show that by building a structured 3D implant with a preperitoneal disk, there is no dislodgement and apparently low to no patient discomfort. The authors acknowledge that this is a very small retrospective study with no control group, but the study revealed lack of long-term complications, such as discomfort, pain and recurrence, which is entirely in line with their theory and results of previous animal studies. In this cohort the elimination of fixation seemed to be a contributing factor to the reduced incidence of chronic pain and discomfort which has been reported in the recent literature as 7.8 and $11 \%$ [17, 18].

The authors fully acknowledge the limitations of this study-specifically that it was retrospective and involved a relatively small number of patients. However, they feel encouraged by the long-term results.

Based upon this initial experience and 3-year data, the implant has been modified to a more industrial design and an expanded prospective single center study and a multicenter trial are being undertaken based upon this protocol. One key area of improvement will be to eliminate subjective pain scores and use recognized pain/comfort scoring systems. The authors intend to perform this in subsequent studies. In summary, this retrospective analysis showed encouraging results in the use of a modified placement technique - based upon dilation of the inguinal defect plus compression of a 3D lamella implant. Short-, medium- and long-term complications were markedly low.
Importantly, the new step of dilation of the orifice does not appear to have any negative effects, especially upon cord structures. In addition to the absence of recurrences, it is noteworthy that the rate of discomfort was extremely low even at 3 years and no patients suffered on chronic postoperative pain.

Conflict of interest A. G declares having received funds in relation with this study. Other authors declare no conflict of interest.

\section{References}

1. Rutkow IM (2003) Demographic and socioeconomic aspects of hernia repair in the United States. Surg Clin North Am 2003(83):1045-1051

2. Bassini E (1889) Nuovo metodo operativo per la cura dell'ernia inguinale. Padua

3. Read RC (2003) Recent advances in the repair of groin herniation. Curr Probl Surg 40(1):13-79

4. Lichtenstein I, Shulman A (1986) Ambulatory outpatient hernia surgery. Including a new concept, introducing tension-free repair. Int Surg 71(1):1-4

5. Amid PK (2004) Causes, prevention, and surgical treatment of postherniorrhaphy neuropathic inguinodynia: triple neurectomy with proximal end implantation. Hernia 8:343-349

6. O'Dwyer PJ, Kingsnorth AN, Mohillo RG, Small PK, Lammers B, Horeysee G (2005) Randomized clinical trial assessing impact of a lightweight or heavyweight on chronic pain after inguinal hernia repair. Br J Surg 92:166-170

7. Rutkow IM, Robbins AW (1995) Mesh plug hernia repair: a follow-up report. Surgery 117:597-598

8. Aasvang E, Kehlet H (2005) Surgical management of chronic pain after inguinal hernia repair. Br J Surg 92:795-801

9. Amato G, Lo Monte AI, Cassata G, Damiano G, Romano G, Bussani R (2012) A new prosthetic implant for inguinal hernia repair: its features in a porcine experimental model. Artif Organs 35(8):E181-E190

10. Amato G, Romano G, Salamone G, Agrusa A, Di Buono G, Gulotta G (2012) Fixation-free inguinal hernia repair, using a dynamic self-retaining implant inducing enhanced biologic response. Results of a midterm follow up. Hernia 16(Suppl 1):S85-S87

11. Amato G, Marasa L, Sciacchitano T, Bell SG, Romano G, Gioviale MC, Lo Monte AI, Romano M (2009) Histological findings of the internal inguinal ring in patients having indirect inguinal hernia. Hernia 13(3):259-262

12. Amato G, Romano G, Salamone G, Agrusa A, Gulotta G, Ober E, Bussani R (2011) Nerve degeneration in inguinal hernia specimens. Hernia 15:53-58

13. Amato G (2010) Reply to the letter: "Histology of the internal inguinal ring: it is really a novelty?". Hernia 14:665-667

14. Amato G, Romano G, Agrusa A, Salamone G, Saladino VA, Silvestri F, Bussani R (2012) Damage to the vascular structures in inguinal hernia specimens. Hernia 16:63-67

15. Amato G, Agrusa A, Romano G, Salamone G, Gulotta G, Silvestri F, Bussani R (2012) Muscle degeneration in inguinal hernia specimens. Hernia 16:327-331

16. Amato G, Sciacchitano T, Bell SG, Romano G, Cocchiara G, Lo Monte AI, Romano M (2009) Sphincter-like motion following mechanical dilation of the rings Internal inguinal hernia during indirect procedures. Hernia 13:67-72 
17. Kim-Fuchs C, Angst E, Vorburger S, Heibling C, Candinas D, Schlumpf R (2012) Prospective randomized trial comparing sutured with sutureless mesh fixation for Lichtenstein hernia repair: long-term results. Hernia 16(1):21-27

18. Negro P, Basile F, Brescia A, Buonanno GM, Campanelli G, Canonico S, Cavalli M, Corrado G, Coscarella G, Di Lorenzo N,
Falletto E, Fei L, Francucci M, Fronticelli Baldelli C, Gaspari AL, Gianetta E, Marvaso A, Palumbo P, Pellegrino N, Piazzai R, Salvi PF, Stabilini C, Zanghì G (2012) Open tension-free Lichtenstein repair of inguinal hernia: use of fibrin glue versus sutures for mesh fixation. Hernia 15(1):7-14 